\title{
Estrés laboral, hábitos alimentarios y estado nutricional antropométrico en trabajadores administrativos y operativos de una clínica privada de Lima, 2018
}

\section{Work stress, eating habits and anthropometric nutritional status in administrative and operational workers of a private clinic in Lima, 2018}

\author{
Juan Manzano Huaracallo ${ }^{1}$
}

\begin{abstract}
RESUMEN
Objetivo: Determinar la relación entre estrés laboral, hábitos alimentarios y estado nutricional antropométrico en los trabajadores administrativos y operativos de una clínica privada de Lima. Metodología: Estudio de enfoque cuantitativo, corte transversal, diseño no experimental, y tipo descriptivo correlacional. Se estudió una muestra de 150 trabajadores adultos, divididos en administrativos y operativos, seleccionados por muestreo no probabilístico intencional. Instrumentos: Se aplicaron encuestas de estrés laboral de la OIT y de hábitos alimentarios, además de evaluación nutricional antropométrica. Resultados: Los participantes mostraron bajos niveles de estrés laboral, siendo $93.3 \%$ para los trabajadores operativos y $94.7 \%$ para los administrativos; también presentaron hábitos alimentarios adecuados en $72 \%$ y $66.7 \%$ respectivamente; finalmente $44 \%$ de trabajadores operativos presentaron sobrepeso, mientras que $60 \%$ de administrativos tenía un IMC normal. Se evidenció que para el personal administrativo existe correlación estadísticamente significativa entre las variables $(p<.05)$, más no para el grupo operativo $(p>.05)$. Conclusión: El estrés laboral y los hábitos alimentarios de los trabajadores administrativos tuvieron un impacto en su estado nutricional, más no en los trabajadores operativos, sin embargo, ambos grupos presentaron bajos niveles de estrés y hábitos alimentarios adecuados; por tanto, los ambientes laborales saludables podrían tienen un impacto positivo en la salud de los mismos.
\end{abstract}

Palabras clave: Estrés laboral, hábitos alimentarios, estado nutricional.

\begin{abstract}
Objective: To determine the relationship between work stress, eating habits and anthropometric nutritional status in administrative and operational workers of a private clinic in Lima. Methodology: Study of quantitative approach, cross-section, non-experimental design, and correlational descriptive type. A sample of 150 adult workers, divided into administrative and operational, Instruments: Was selected by intentional non-probabilistic sampling. Surveys of ILO labor stress and eating habits were applied, in addition to anthropometric nutritional evaluation. Results: Participants showed low levels of work stress, with $93.3 \%$ for operational workers and $94.7 \%$ for administrative workers; they also presented adequate eating habits in $72 \%$ and $66.7 \%$ respectively; Finally, $44 \%$ of operational workers were overweight, while $60 \%$ of administrative workers had a normal BMI. It was shown that for the administrative staff there is a statistically significant correlation between the variables $(p<.05)$, but not for the operating group $(p>.05)$. Conclusion: work stress and eating habits of administrative workers had an impact on their nutritional status, but not on operational workers, however, both groups had low levels of stress and adequate eating habits; Therefore, healthy work environments could have a positive impact on their health.
\end{abstract}

Keywords: Work stress, eating habits, nutritional status 


\section{INTRODUCCIÓN}

El estado nutricional es uno de los problemas que más aquejan a la población mundial; en la actualidad diagnósticos como el sobrepeso y obesidad han sido ampliamente estudiados en diversos países; estimando que en el mundo una de cada tres personas sufre de desnutrición, sobrepeso u obesidad, tal es así que en el 2014 la Organización Mundial de la Salud mencionó que "más de 1900 millones de personas mayores de 18 años tenían sobrepeso, y entre ellos 600 millones eran obesos".

De la Cruz (2016) menciona que entre el 2014 y 2015 el $23 \%$ de la población adulta mayor a 20 años de América Latina y el Caribe era obesa, lo que significa que 1 de cada 4 habitantes de esta región se encuentra afectada. Los países del Caribe como San Cristóbal y Nieves $(40,9 \%)$, Bahamas (35\%), y Barbados $(33,4 \%)$ tienen las mayores prevalencias de obesidad en la región; en Centro américa, México cuenta con la mayor prevalencia de obesidad (32,8\%); mientras que en Sudamérica se presenta mayor porcentaje de adultos obesos en Venezuela (30,8\%), Argentina $(29,4 \%)$, Chile $(29,1 \%)$, Panamá $(25,8 \%)$, Nicaragua $(24,2 \%)$, Ecuador (22,0\%), República Dominicana $(21,9 \%)$, Guatemala $(20,7)$, Cuba $(20,5)$, Brasil $(19,5)$, Paraguay $(19,2)$, Colombia (18,1\%), Perú (16,5\%). (FAO, 2015).

En el Perú, según el informe técnico (2013 - 2014) del Ministerio de Salud (MINSA), según Mamani y Alvarez (2015) el estado nutricional según IMC de los adultos se distribuye en: delgadez $0.6 \%$, normal $29.5 \%$, sobrepeso $46.1 \%$, obesidad I en $18.6 \%$, obesidad II en $4.2 \%$ y obesidad III en $1 \%$, mientras que demográficamente el sobrepeso y obesidad se distribuye en: sierra centro (41.3\%), sierra norte $(42.6 \%)$, sierra sur $(44.2 \%)$, costa sur $(43,1 \%)$, costa centro $(48,7 \%)$, costa norte $(52.2 \%)$, selva (44.5\%) y Lima metropolitana (46.2\%).

Así también, diversos estudios muestran la prevalencia del sobrepeso y obesidad en trabajadores del área administrativa de diversas empresas peruanas; dentro de ellas tenemos la investigación de Rosas, Lama, Llanos, Dunstan (2002) donde se muestra una prevalencia. de $64.7 \%$ en el personal administrativo de una institución estatal, por su parte Sanabria et al. (2014) encontraron una prevalencia de $64.0 \%$ en los trabajadores administrativos de la Dirección Regional de Salud de Lima, Puescas, Cabrera y Diaz (2012) reportaron una prevalecía de $64.7 \%$ en trabajadores administrativos del área de salud, Arbulú (2014) reportó una prevalencia de $72.5 \%$ en trabajadores de la Universidad Peruana de Ciencias Aplicadas, asimismo
Allpas et all (2016) revelaron una prevalencia de sobrepeso y obesidad del $70.2 \%$ de trabajadores de una empresa peruana y Paz (2014) encontró un a prevalencia de $87.6 \%$ en trabajadores administrativos de una empresa privada peruana, Por otro lado, en estudios sobre personal operático encontramos los siguientes resultados; SEDAPAL (2013) reportó que el $81.5 \%$ de sus trabajadores operativos muestran prevalencia de sobrepeso y obesidad, asimismo Allpas et al (2016) encontraron una prevalecía de $73.4 \%$ en sus trabajadores, Velásquez, Palomino y Ticse (2013) reportaron una prevalencia de $68.3 \%$ y Ramos (2017) encontró una prevalencia de $80 \%$ .en el personal operático de una empresa de transporte arequipeña.

Potocka, Moscicka (2011) y Yau, Potenza, (2013) posturaron que el sobrepeso y obesidad en la población económicamente activa está vinculados con diversos factores, entre los cuales está el estrés y lo hábitos alimentarios incluso estudios sugieren que el estrés laboral, aumenta la probabilidad de aumento de peso entre los hombres que tiene un IMC mayor de $27 \mathrm{~kg} / \mathrm{m} 2$ y predice la pérdida de peso entre hombres con un IMC menor a $22 \mathrm{~kg} / \mathrm{m} 2$ (Kivimäki et al 2006). Por tanto, nace la pregunta si realmente existe relación entre el estrés laboral, hábitos alimentarios y el estado nutricional antropométrico en trabajadores con actividades administrativas y operativas, que son los grupos más diferenciados dentro de una empresa.

\section{METODOLOGÍA}

Este estudio tiene un enfoque cuantitativo porque los datos se analizarán estadísticamente; de diseño no experimental pues no se manipularán las variables; de corte transversal porque se tomará los datos solo en un momento determinado; y de tipo correlacional porque se busca determinar la relación entre estrés laboral, hábitos alimentarios y estado nutricional.

La población estuvo conformada por 245 trabajadores, de los cuales 140 son trabajadores operativos y 105 trabajadores administrativos siendo la muestra representada por 150 personas adultas de ambos sexos seleccionados mediante muestreo no probabilístico a conveniencia del investigador, representando el $61.2 \%$ de la población total.

La recolección de los datos, se realizó mediante la aplicación del cuestionario de la Escala de Estrés Laboral de la OIT-OMS adaptada por Suarez (2013). el cuestionario de hábitos 
alimentarios elaborado por el investigador y la toma de datos antropométricos (IMC y perímetro abdominal).

La Escala de Estrés Laboral de la OIT-OMS adaptada por Suarez (2013), tiene confiabilidad por el método de consistencia interna (Alfa de Cronbach $=0.972$ ) y tiene validez de constructo y contenido. La escala evalúa siete dimensiones relacionadas al estrés laboral las cuales son: Clima, estructura, territorio, tecnología, influencia del líder, falta de cohesión y respaldo del grupo a nivel organizacional. Si la puntuación resulta ser menor de 90.2 puntos se clasificará como bajo nivel de estrés; si es de 90.3 - 117.2 puntos sería nivel intermedio de estrés; de 117.3 - 153.2 puntos se denominaría como estrés; y puntajes mayores a 153.3 denotarán un alto nivel de estrés.

El cuestionario de hábitos alimentarios fue validado por jueces expertos (3 magísteres y 2 licenciados en Nutrición), y por prueba piloto en 90 trabajadores, con una confiabilidad de alfa de cronbach de 0,782. El cuestionario consta de 47 ítems y 6 dimensiones: número de comidas, horarios, frecuencia de consumo, cantidades, tipo de preparaciones y consumo de alimentos de alto valor calórico.

Para determinar el estado nutricional antropométrico se evaluó el IMC y perímetro abdominal de cada participante y se registró los datos en una ficha de evaluación nutricional.

Finalmente, el registro y ordenamiento de los datos se realizaron con el programa Microsoft Excel en su versión 2016, mientras que, para el procesamiento y análisis de los datos se utilizó el programa SPSS, versión 23; aplicando en el análisis descriptivo tablas de frecuencia y para el análisis de relación la prueba estadística Rho de Spearman.

Antes de ejecutar la investigación se procedió a solicitar el permiso correspondiente al departamento de docencia e investigación de la clínica en mención y de acuerdo con las normas éticas se aseguró la participación voluntaria y anónima de sus trabajadores, luego cada participante firmó un consentimiento informado.

\section{RESULTADOS}

En la tabla 1 se observa que para el grupo de personal operativo no existe relación estadísticamente significativa entre el estrés, hábitos alimentarios y estado nutricional ( $p>.05$ ), mientras que, para el grupo administrativo, existe una relación estadísticamente significativa entre estrés laboral y hábitos alimentarios $(p=0,003)$ de forma inversamente proporcional.

Tabla 1

Análisis de relación entre el estrés, hábitos alimentarios e IMC.

\begin{tabular}{|c|c|c|c|c|}
\hline \multicolumn{4}{|c|}{ Grupo } & \multirow{2}{*}{$\begin{array}{l}\text { Hábitos Alimentarios } \\
-0.068\end{array}$} \\
\hline \multirow{17}{*}{$\begin{array}{l}\text { Rho de } \\
\text { Spearman }\end{array}$} & \multirow{8}{*}{$\begin{array}{l}\text { Operativo } \\
(75)\end{array}$} & Estrés & Coeficiente de correlación & \\
\hline & & $(75)$ & $\mathrm{p}$ & 0.561 \\
\hline & & \multirow{3}{*}{ Estrés } & & IMC \\
\hline & & & Coeficiente de correlación & -0.057 \\
\hline & & & $\mathrm{p}$ & 0.628 \\
\hline & & \multirow{3}{*}{ Hábitos Alimentarios } & & IMC \\
\hline & & & Coeficiente de correlación & -0.044 \\
\hline & & & $\mathrm{p}$ & 0.707 \\
\hline & \multirow{9}{*}{$\begin{array}{l}\text { Administrativo } \\
(75)\end{array}$} & \multirow{3}{*}{ Estrés } & & Hábitos Alimentarios \\
\hline & & & Coeficiente de correlación & -0.336 \\
\hline & & & $\mathrm{p}$ & 0.003 \\
\hline & & \multirow{3}{*}{ Estrés } & & IMC \\
\hline & & & Coeficiente de correlación & 0,330 \\
\hline & & & $\mathrm{p}$ & 0.004 \\
\hline & & \multirow{3}{*}{ Hábitos Alimentarios } & & IMC \\
\hline & & & Coeficiente de correlación & $-0,131$ \\
\hline & & & $\mathrm{P}$ & 0.264 \\
\hline
\end{tabular}


La tabla 2 muestra que el $94.7 \%$ de trabajadores administrativos tienen un bajo nivel de estrés, $2.7 \%$ nivel intermedio de estrés y estrés laboral, respectivamente. Estos datos se profundizan con los resultados de las dimensiones del estrés evaluadas por la OIT, que evidencia que el personal administrativo presenta en promedio mayor nivel de estrés en la dimensión de uso de tecnología en $14 \%$ e influencia del líder en $9 \%$; mientras que las dimensiones con menor promedio de estrés laboral fueron el territorio o ambiente organizacional en $4 \%$ y la falta de cohesión o trabajo en equipo en $4 \%$.

Tabla 2

Determinación del nivel de estrés laboral de los trabajadores en los administrativos y operativos.

\begin{tabular}{lllll} 
& & & Operativo & Administrativo \\
& Bajo nivel de estrés & $\mathrm{N}$ & 70 & 71 \\
& & $\%$ & $93.3 \%$ & $94.7 \%$ \\
Estrés & Nivel intermedio & $\mathrm{N}$ & 4 & 2 \\
& & $\%$ & $5.3 \%$ & $2.7 \%$ \\
& Estrés & $\mathrm{N}$ & 1 & 2 \\
& & $\%$ & $1.3 \%$ & $2.7 \%$ \\
& Total & $\mathrm{N}$ & 75 & 75 \\
& & $\%$ & $100.0 \%$ & $100.0 \%$ \\
\hline
\end{tabular}

Los resultados expresados en la tabla 3 muestran mayor prevalencia de hábitos alimentarios adecuados, siendo $72 \%$ para los trabajadores operativos y $66.7 \%$ para los administrativos. Sin embargo, según indica la tabla 1, existe una correlación inversamente proporcional entre los hábitos alimentarios y los niveles de estrés laboral en el personal administrativo, por tanto, es relevante destacar que solo el $33.3 \%$ del personal administrativo presenta hábitos alimentarios inadecuados, siendo mayor que en los trabajadores operativos con $28 \%$.

Tabla 3

Determinación de los hábitos alimentarios de los trabajadores administrativos y operativos

\begin{tabular}{lllll} 
& & & Operativo & Administrativo \\
& Inadecuado & $\mathrm{N}$ & 21 & 25 \\
Hábitos & $\%$ & $28 \%$ & $33.3 \%$ \\
alimentarios & Adecuado & $\mathrm{N}$ & 54 & 50 \\
& & $\%$ & $72 \%$ & $66.7 \%$ \\
& Total & $\mathrm{n}$ & 75 & 75 \\
& & $\%$ & $100.0 \%$ & $100.0 \%$ \\
\hline
\end{tabular}

Según la tabla 4 , se observa que $69.3 \%$ de los trabajadores operativos presenta sobrepeso u obesidad y sólo $30.7 \%$ tiene un IMC normal. En cambio, para el personal administrativo, $60 \%$ presenta un diagnóstico normal y $40 \%$ presenta sobrepeso y obesidad. 
Tabla 4

Determinación del IMC de los trabajadores administrativos y operativos

\begin{tabular}{|c|c|c|c|c|}
\hline & & & Operativo & Administrativo \\
\hline \multirow{8}{*}{ IMC } & \multirow{2}{*}{ Normal } & $N$ & 23 & 45 \\
\hline & & $\%$ & $30.7 \%$ & $60.0 \%$ \\
\hline & \multirow{2}{*}{ Sobrepeso } & $\mathrm{N}$ & 33 & 22 \\
\hline & & $\%$ & $44.0 \%$ & $29.3 \%$ \\
\hline & \multirow{2}{*}{ Obesidad } & $\mathrm{N}$ & 19 & 8 \\
\hline & & $\%$ & $25.3 \%$ & $10.7 \%$ \\
\hline & \multirow{2}{*}{ Total } & $\mathrm{N}$ & 75 & 75 \\
\hline & & $\%$ & $100.0 \%$ & $100.0 \%$ \\
\hline
\end{tabular}

\section{DISCUSIÓN}

En la tabla 1 se observa que no existe estadísticamente significativa entre el estrés, hábitos alimentarios y estado nutricional en el personal operativo, sin embargo, existe una relación estadísticamente significativa entre estrés laboral y hábitos alimentarios. Esto se debe a que la mayoría del personal administrativo presenta bajo nivel de estrés laboral $(94,7 \%)$ según tabla 2 y hábitos alimentarios adecuados (66,7\%), según tabla 3 . Del mismo modo, Anchante y Zevallos (2017), en su estudio realizado en Perú, demostraron que existe correlación significativa entre hábitos alimentarios y estrés laboral $(p=0,000)$ en enfermeras de las áreas críticas del Instituto Nacional de Salud del Niño de Lima. Por otro lado, los resultados que comparan el estrés laboral con IMC, señalan que para el mismo grupo existe correlación estadísticamente significativa $(p=0,004)$, a pesar de que solo $5.4 \%$ del grupo de administrativos presenta estrés laboral y el $40 \%$ de los mismos presenta sobrepeso y obesidad, lo que indica que el estrés es un factor de predisposición a un incremento del IMC. También, Kouvonen et al (2005) realizaron un estudio en trabajadores municipales y hospitales de Finlandia que demostró la correlación significativa de un mayor IMC con tensión laboral $(p$.001) y un mayor desequilibrio esfuerzo-recompensa ( $p$ .001). Asimismo, Nishitani y Sakakibara (2006) analizaron la relación de la obesidad con el estrés laboral y el comportamiento alimentario en trabajadores japoneses varones de una empresa manufacturera, donde se evidenció una correlación significativa de la obesidad con respuestas de estrés psicológico de tensión / ansiedad relacionados con la demanda laboral de carga de trabajo y conflicto interpersonal $(P<0.01)$. Por consiguiente, es evidente que el estrés laboral es una condición influyente en el desarrollo de comportamiento alimentarios inadecuados, que finalmente se traducen en un incremento del IMC.

En la tabla 2 se evidencia que el $94.7 \%$ de trabajadores administrativos tienen un bajo nivel de estrés, $2.7 \%$ nivel intermedio de estrés y estrés laboral. Del mismo modo, Hilario y Quispe (2016) realizaron un estudio sobre los niveles de estrés y clima laboral en los colaboradores de una empresa privada de Lima, con la intención de determinar la prevalencia de estrés encontrando, donde $87,50 \%$ de los colaboradores presentaron bajo nivel de estrés, $7,74 \%$ un nivel intermedio y $4,76 \%$ estrés, además las dimensiones con más alto porcentaje fueron clima organizacional y falta de cohesión con $9.61 \%$ cada una, mientras que la dimensión de territorio organizacional sólo $6.39 \%$, siendo el más bajo. Por el contrario, Perales, Chue, Pdilla , Barahona (2011), en un estudio realizado en Lima sobre trabajadores del poder judicial y ministerio público, hallaron una prevalencia de estrés laboral de $33,7 \%$ en general; estos resultados además indican que el estrés laboral fue mayor en varones con 43,9 \% y en trabajadores de 5 a 10 años de antigüedad en $43,3 \%$, identificando que un mayor nivel de estrés laboral está relacionado con un mayor nivel de depresión y ansiedad. Por lo tanto, en base a los resultados y estudios comparados, se evidencia que los trabajadores de cada empresa reaccionan al estrés laboral en diferentes niveles con énfasis en distintas dimensiones; lo que finalmente tiene un efecto negativo en la salud general de los trabajadores, el cual se puede expresar en sobrepeso, obesidad y enfermedades crónicas no trasmisibles. 
En la tabla 3 se muestra la prevalencia de hábitos alimentarios donde el $72 \%$ de los trabajadores operativos y $66.7 \%$ de los trabajadores administrativos tienen una adecuados prevalencia de hábitos alimenticios.

Las dimensiones que más destacan entre los trabajadores administrativos son el consumo de alimentos de alto valor calórico con $76 \%$, una inadecuada frecuencia y condición de consumo de alimentos saludables con $56 \%$ y con menor prevalencia está el consumo de alimentos en preparaciones poco saludables en $11 \%$. En cambio, Arbulú (2014), al investigar acerca del sobrepeso y estilo de vida de trabajadores administrativos de una empresa privada de Lima, observó que el $37,4 \%$ de población estudiada tenía un elevado consumo de kilocalorías por día, el cual se expresaba en elevado consumo de carbohidratos en $57,2 \%$, insuficiente consumo de fibra en $99,2 \%$ y el consumo diario de frutas en $38,2 \%$. Esto probablemente se debe a la diferencia de los entornos de trabajo, carga laboral, acceso a alimentos no saludables, entre otros. Sin embargo, los trabajadores operativos también presentan hábitos alimentarios inadecuados, como lo menciona Toledo (2014), en su estudio realizado a trabajadores de una empresa de transporte de Lima, demostrando que el $53 \%$ presenta hábitos alimentarios no saludables, $46 \%$ hábitos poco saludables y solo el $1 \%$ tiene hábitos alimentarios saludables. También, Orihuela y Venegas (2013), hallaron que entre el personal operativo de empresas de transporte limeños predominaban hábitos alimentarios inadecuados, siendo que 93,3\% consumen más de 12 porciones de carbohidratos; $82.5 \%$ consume gaseosas 3 a 6 veces por semana; $50 \%$ consume menos de 1 porción de frutas al día y el $90 \%$ de 1 a 2 porciones de verduras solo una vez por semana, siendo el consumo menor a lo recomendado. Estos datos muestran la diferencia de resultados en diversas empresas, lo que implica que los hábitos alimentarios están relacionados a diversos factores, siendo uno de ellos el estrés laboral.

En la Tabla 4 se evidencia que solamente el $30 \%$ de tiene un IMC normal, siendo que el $69.3 \%$ de los trabajadores operativos presenta sobrepeso u obesidad. En cambio, para el personal administrativo, $60 \%$ presenta un diagnóstico normal y $40 \%$ presenta sobrepeso y obesidad.
Sin embargo, según la tabla 1 se demostró que el índice de masa corporal de los trabajadores administrativos tuvo relación significativa directamente proporcional al estrés laboral, lo que significa que, a mayor grado de estrés, se presenta mayor IMC. Además, el $58.7 \%$ de los trabajadores operativos y $42 \%$ de los administrativos presentan valores elevados de perímetro abdominal, lo que implica mayor riesgo cardiovascular y desarrollo de enfermedades crónicas no transmisibles. En cambio, Rosas, Lama, Llanos, Dunstan (2002) en un estudio realizado en una institución estatal de Lima a trabajadores administrativos, hallaron que el $46.8 \%$ de población tenía sobrepeso y $17.9 \%$ obesidad, sumando un $64.7 \%$ de exceso de peso, y sólo $35.3 \%$ presentaron un IMC normal. También, Arbulú (2014), reporta un 51.9\% de sobrepeso y $20.6 \%$ de obesidad, haciendo un total de $72.5 \%$ de exceso de peso en su población de estudio y sólo un $27.5 \%$ con un peso normal, además un $26 \%$ de prevalencia de riesgo cardiovascular, es decir un perímetro abdominal alto. Los resultados hallados en este estudio muestran que los trabajadores administrativos presentan una menor prevalencia de obesidad y sobrepeso a diferencia de otros estudios en empresas con el mismo tipo de trabajadores, esto puede deberse a diversos factores como mayor nivel educativo, hábitos alimentarios más saludables y un mejor clima laboral.

\section{CONCLUSIÓN}

En base al análisis realizado se concluye que existe relación significativa entre el estrés laboral y hábitos alimentarios para el personal administrativo, como también entre estrés laboral y estado nutricional antropométrico, más no se encontró correlación entre las variables para el personal operativo.

En relación al nivel de estrés laboral de los trabajadores administrativos y operativos se halló que la mayoría de trabajadores tenía un nivel bajo de estrés laboral, el cual se centraba en el uso de la tecnología e influencia del líder.

Acerca de los hábitos alimentarios de los trabajadores, existe mayor prevalencia de hábitos alimentarios adecuados, encontrándose relación con el estrés laboral solo en el personal administrativo. 
Finalmente, en referencia al estado nutricional antropométrico se evidencia mayor prevalencia de sobrepeso y obesidad en el personal operativo.

Declaración de financiamiento y de conflictos de interés:

El estudio fue financiado por los autores, quienes declaran no tener algún tipo de conflicto de interés en la investigación realizada.

\section{Correspondencia}

Juan Manzano Huaracallo

Correo electrónico:

jluisl10@hotmail.com

\section{REFERENCIAS BIBLIOGRÁFICAS}

OMS. (2016).Centro de Prensa. Obesidad y sobrepeso. Recuperado de http://www.who.int/ mediacentre/factsheets/fs311/es/

De La Cruz, E. (2016). La transición nutricional. Abordaje desde de las políticas públicas en América Latina. Red Revistas Científicas de América Latina, el Caribe, España y Portugal, 32(11), 302-379. Recuperado de: http://www. redalyc.org/pdf/310/31048902022.pdf

FAO. (2015). Panorama de la Inseguridad Alimentaria en América Latina y el Caribe. Organización de las Naciones Unidas para la Agricultura y la Alimentación, 2-78. Recuperado de: http://www. fao.org/3/a-i4636s.pdf

MINSA. (2015). Estado nutricional por etapas de vida en la población peruana; 2013-2014. Recuperado de https://web.ins.gob.pe/sites/default/files/ Archivos/cenan/van/vigilacia_poblacion/VIN_ ENAHO_etapas_de_vida_2013-2014.pdf

Rosas, A., Lama, G., Llanos, F., Dunstan, J. (2002). Prevalencia de obesidad e hipercolesterolemia en trabajadores de una institución estatal de Lima - Perú. Rev Peru Med Exp Salud Publica. 2002;19(2):87-92.Recuperado de: http:// www.scielo.org.pe/scielo.php? pid=S172646342002000200007\&script=sci_abstract

Sanabria, H., Tarqui, C., Portugal, W., Pereyra, H., Mamani, L. (2014) . Nivel de actividad física en los trabajadores de una Dirección Regional de Salud de Lima, Perú. Revista de Salud Pública, 16(1), 53-62. Recuperado de https://revistas. unal.edu.co/index.php/revsaludpublica/article/ view/38672/61974
Puescas, P., Cabrera, J., Díaz, C. (2012). Factores de riesgo de enfermedad cardiovascular, un problema en trabajadores de la salud. Acta Médica Peruana, 29(1). Recuperado de http:// www.scielo.org.pe/scielo.php?script=sci_arttext\& pid=S1728-59172012000100004

Arbulú, X. (2014). Sobrepeso y estilo de vida de trabajadores administrativos de una empresa privada de Lima. (Tesis de licenciatura, Universidad Peruana de Ciencias Aplicadas). Recuperado de https://repositorioacademico. upc.edu.pe/bitstream/handle/10757/347100/ Tes is $\% 20$ Arbul \% c $3 \%$ b a \% $20 \mathrm{Pizarro}$. pdf?sequence $=1 \&$ isAllowed $=y$

Allpas, H., Rodriguez, O., Lezama, J., Raraz, O. (2016). Enfermedades del trabajador en una empresa peruana en aplicación de la ley de seguridad y salud en el trabajo. Horizonte Médico, 16(1), 48-54. Recuperado de http://www.scielo. org.pe/pdf/hm/v16n1/a07v16n1.pdf

Castillo, E. (2014). Alteraciones metabólicas en los trabajadores de una empresa pública en Lima, y la relación con medidas antropométricas. (Tesis de licenciatura, Universidad Peruana de Ciencias Aplicadas). Recuperado de https:// repositorioacademico.upc.edu.pe/bitstream/ handle/10757/338145/TESIS\%20TERESA $\% 20$ CASTILLO.pdf?sequence $=1 \&$ isAllowed $=y$

Servicio de Agua Potable y Alcantarillado (SEDAPAL). (2013). Estado Nutricional de los trabajadores de una empresa de saneamiento de Lima, Perú.

Velasquez, C., Palomino, J., Ticse, R. (2017). Relación entre el estado nutricional y los grados de ausentismo laboral en trabajadores de dos empresas peruanas. Acta Medica, 34(1), 6-15. Recuperado de http://www.scielo.org.pe/pdf/ amp/v34n1/a02v34n1.pdf

Ramos, M. (2017). Valoración de sobrepeso y obesidad según el puesto laboral, en trabajadores de transporte público de la ciudad de Arequipa - Perú. (Tesis de licenciatura, Universidad Católica de Santa María). Recuperado de https://alicia.concytec.gob.pe/vufind/Record/UC SM_5bda52ed81a5dfd24c7459a623269781/ Description\#tabnav

Potocka, A., Mościcka, A. (2011). Occupational stress, coping styles and eating habits among Polish employees. Med Pr, 62(4), 377-88. Recuperado de: https://www.ncbi.nlm.nih.gov/ pubmed/21995107

Kim, H., Kim, J. (2009). Relationship between Stress and Eating Habits of Adults in Ulsan, Korean Nutrition Society, 42(6), 536-46. Recuperado de https://synapse.koreamed.org/Synapse/Data/ PDFData/0124KJN/kjn-42-536.pdf 
Yau, Y., Potenza, M. (2013). Stress and eating behaviors. Minerva Endocrinologica 38(3), 25567. Recuperado de https://www.minervamedica. it/en/journals/minerva-endocrinologica/article. php?cod=R07Y2013N03A0255

Kivimäki, M., Head, J., Ferrie, J., Shipley, M., Brunner, E., Vahtera, J., Marmot, M. (2006). Work stress, weight gain and weight loss: evidence for bidirectional effects of job strain on body mass index in the Whitehall II study. Int J Obes, 30(6), 982-7. Recuperado de https://www.ncbi.nlm.nih. gov/pubmed/16418750

Suarez Á. (2013). Adaptación de la Escala de estrés Laboral de la OIT-OMS en trabajadores de 25 a 35 años de edad de un Contact Center de Lima. Revista PsiqueMag, 2(1), 33-50. Recuperado de http://revistas.ucv.edu.pe/index.php/psiquemag/ issue/download/208/Psiquemag\%202013-3

Anchante, M., Zevallos, K. (2018). Estilos de vida saludable y estrés laboral en enfermeras de áreas críticas del Instituto Nacional de Salud del Niño, Lima, 2017. (Tesis de maestría; Universidad Cesar Vallejo). Recuperado de http://repositorio. ucv.edu.pe/bitstream/handle/UCV/15060/ Anchante_SMM.pdf?sequence=1\&isAllowed=y

Kouvone, A., Kivimäki, M., Cox, S., Cox, T., Vahtera, J. (2005). Relationship between work stress and body mass index among 45,810 female and male employees;. Psychosom Med, 67(4), 577-83. Recuperado de http://www.ncbi.nlm.nih.gov/ pubmed/16046370

Nishitani, N., Sakakibara, H. (2006). Relationship of obesity to job stress and eating behavior in male Japanese workers. Int J Obes, 30(3), 528-33. Recuperado de http://www.ncbi.nlm.nih.gov/ pubmed/16247505
Hilari, J., Quispe, S. (2016). Niveles de estrés y clima laboral en los colaboradores de una empresa privada de Lima. (Tesis de licencitura, Universidad Autónonma del Perú). Recuperado de http://repositorio.autonoma.edu.pe/handle/ AUTONOMA/366

Perales, A., Chue, H., Padilla, A., Barahona, L. (2011). Estrés, ansiedad y depresión en magistrados de Lima, Perú. Revista Peruna de Medicina Experimental y Salud Pública, 28(4), 581-8. Recuperado de: http://www. scielo.org.pe/scielo.php?script=sci_arttext\&pid =S1726-46342011000400002

Toledo, H. (2016). Hábitos alimentarios y consumo alimentario en relación al estado nutricional de los choferes de la empresa Virgen de Fátima de la ciudad de Puno y Juliaca - 2014. (Tesis de licenciatura, Universidad Nacional del Altiplano) Recuperado de http://tesis.unap.edu. pe/bitstream/handle/UNAP/2154/Toledo_Zela_ Hely_Liliana.pdf?sequence=1\&isAllowe

Orihuela, L., Venegas, D. (2013). Prevalencia de Sobrepeso y Obesidad relacionado a los hábitos alimentarios en choferes de las empresas de Transporte de Carga Pesada" Baslit" y urbano Señor de Muruhuay; Lima 2013. (Tesis de licenciatira, Universidad Peruana Unión). Recuperado de https://repositorio.upeu.edu. pe/bitstream/handle/UPEU/616/Lizbet_Tesis bachiller_2013.pdf?sequence=1\&isAllowed=y

Recibido: 10 de mayo Aceptado: 2 de setiembre 